\title{
Experimental study of nonlinear interaction of plasma flow with charged thin current sheets: 1. Boundary structure and motion
}

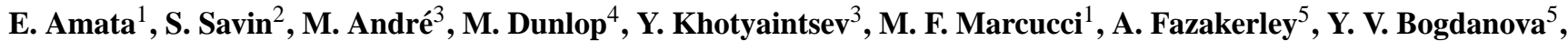 \\ P. M. E. Décréau ${ }^{6}$, J. L. Rauch ${ }^{6}$, J. G. Trotignon ${ }^{6}$, A. Skalsky ${ }^{2}$, S. Romanov ${ }^{2}$, J. Buechner ${ }^{7}$, J. Blecki ${ }^{8}$, and H. Rème ${ }^{9}$ \\ ${ }^{1}$ Istituto di Fisica dello Spazio Interplanetario, INAF, Via del fosso del cavaliere 100, 00133 Roma, Italy \\ ${ }^{2}$ Space Research Institute, 117997, Profsoyuznaya 84/32, Moscow, Russia \\ ${ }^{3}$ Swedish Institute of Space Physics, Uppsala, Sweden \\ ${ }^{4}$ Space Science and Technology Department, Rutherford Appleton Laboratory, UK \\ ${ }^{5}$ Mullard Space Science Laboratory, Holmbury St Mary, Surrey, UK \\ ${ }^{6}$ Laboratoire de Physique et Chimie, de l'Environnement, CNRS, Orléans, France, France \\ ${ }^{7}$ Max-Planck-Institut fur Sonnensystemforschung, Katlenburg-Lindau, Germany \\ ${ }^{8}$ Space Research Center, Polish Academy of Sciences, Warsaw, Poland \\ ${ }^{9}$ Centre d'Etude Spatiale de Rayonnement, CNRS/UPS/OMP, Toulouse, France
}

Received: 3 May 2005 - Revised: 22 May 2006 - Accepted: 22 May 2006 - Published: 10 August 2006

Part of Special Issue "Nonlinear and multiscale phenomena in space plasmas"

\begin{abstract}
We study plasma transport at a thin magnetopause (MP), described hereafter as a thin current sheet (TCS), observed by Cluster at the southern cusp on 13 February 2001 around 20:01 UT. The Cluster observations generally agree with the predictions of the Gas Dynamic Convection Field (GDCF) model in the magnetosheath (MSH) up to the MSH boundary layer, where significant differences are seen. We find for the MP a normal roughly along the GSE $\mathrm{x}$ axis, which implies a clear departure from the local average MP normal, a $\sim 90 \mathrm{~km}$ thickness and an outward speed of $35 \mathrm{~km} / \mathrm{s}$. Two populations are identified in the MSH boundary layer: the first one roughly perpendicular to the MSH magnetic field, which we interpret as the "incident" MSH plasma, the second one mostly parallel to $\mathbf{B}$. Just after the MP crossing a velocity jet is observed with a peak speed of $240 \mathrm{~km} / \mathrm{s}$, perpendicular to $\mathbf{B}$, with $M_{A}=3$ and $\beta>10$ (peak value 23 ). The magnetic field clock angle rotates by $70^{\circ}$ across the MP. $E_{x}$ is the main electric field component on both sides of the MP, displaying a bipolar signature, positive on the MSH side and negative on the opposite side, corresponding to a $\sim 300 \mathrm{~V}$ electric potential jump across the TCS. The $E \times B$ velocity generally coincides with the perpendicular velocity measured by CIS; however, in the speed jet a difference between the two is observed, which suggests the need for an extra flow source. We propose that the MP TCS can act locally as an obstacle for low-energy ions $(<350 \mathrm{eV})$,
\end{abstract}

being transparent for ions with larger gyroradius. As a result, the penetration of plasma by finite gyroradius is considered as a possible source for the jet. The role of reconnection is briefly discussed. The electrodynamics of the TCS along with mass and momentum transfer across it are further discussed in the companion paper by Savin et al. (2006).

\section{Introduction}

The magnetosheath/cusp transition was first investigated over thirty years ago through the HEOS, Hawkeye, Prognoz8 and Prognoz-10 missions (e.g. see Paschmann et al., 1976; Haerendel et al., 1978; Farrell and Van Allen, 1990; Vaisberg et al., 1983; Klimov et al., 1986; Blecki et al., 1998; Kessel et al., 1996; Eastman et al., 2000; Dunlop et al., 2000), and more recently re-explored with the Polar mission (e.g. Grande et al., 1997; Russell, 2000; Scudder et al., 2002; Fritz et al., 2003) as well as the Interball mission (e.g. Fedorov et al., 2000; Dubinin et al., 2002; Savin et al., 1998, 2004a, b and 2005a, b;); finally, the cusp bibliography based on Cluster data is now large enouth (see Lavraud et al., 2002 and 2005; Cargill et al., 2004; Dunlop et al., 2005 and references therein).

Correspondence to: E. Amata

(emanno.amata@ifsi-roma.inaf.it)

Published by Copernicus GmbH on behalf of the European Geosciences Union and the American Geophysical Union. 


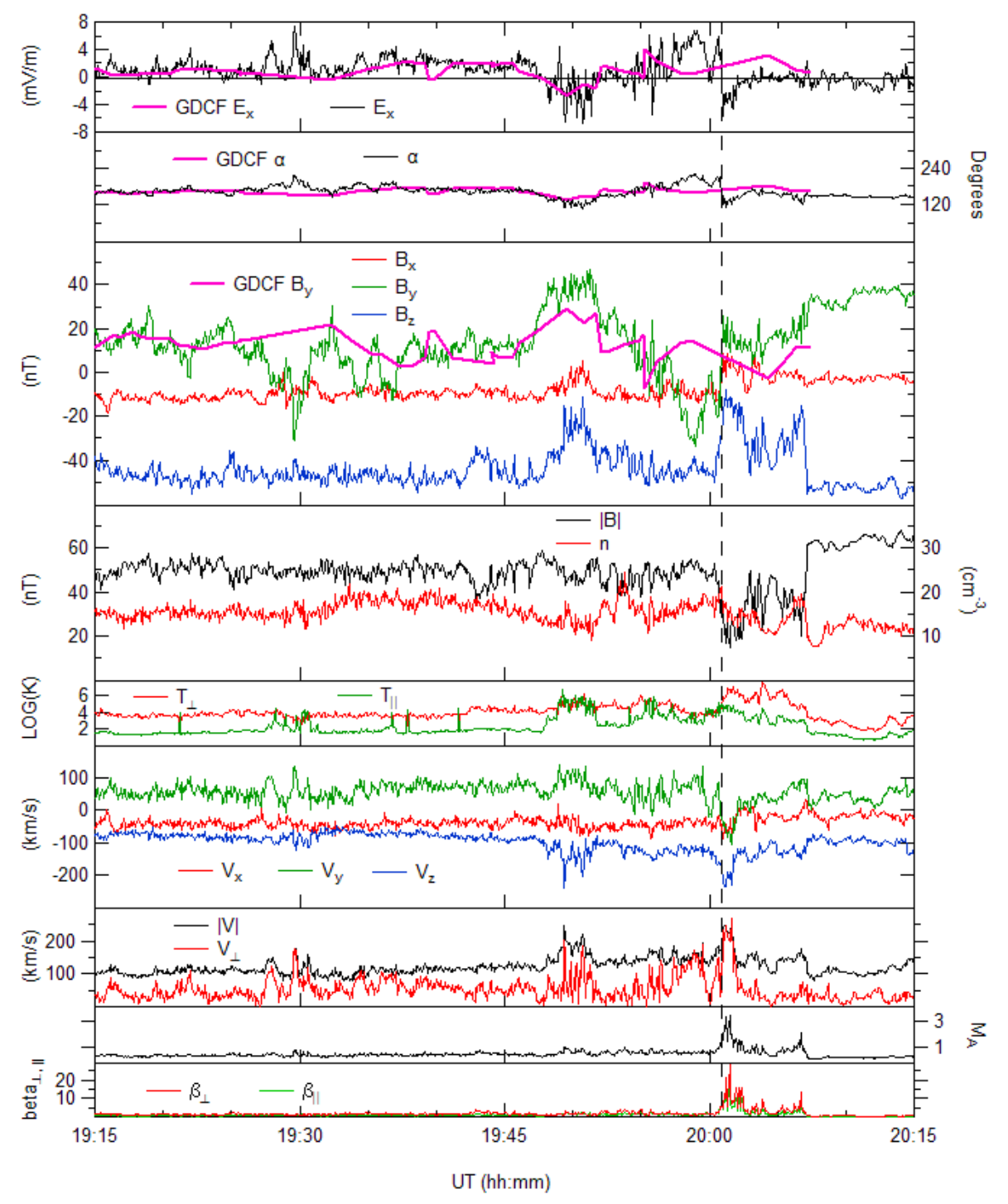

Fig. 1. Data from 19:15 to 20:15 UT on 13 February 2001. From top to bottom: x GSM component of the electric field, as measured by SC1 and as calculated through the GDCF model; magnetic field GSM clock angle as calculated from SC1 data and from GDCF; SC1 GSM three magnetic field components together with the GDCF GSM $B_{y}$; total magnetic field intensity and CIS-HIA ion number density, corrected by a 1.38 factor obtained by intercalibrating the CIS-HIA data with the Whisper data; parallel and perpendicular CIS-HIA temperatures; three GSM components of the CIS-HIA proton velocity; total speed and total perpendicular speed; Alfvén Mach number; parallel and perpendicular plasma beta.

The cusps are believed to be the main places of transport of plasma into the magnetosphere and therefore contain modified MSH plasma. Their extent and location are known to respond to Interplanetary Magnetic Field (IMF) and to solar wind pressure (e.g. Frank, 1971; Newell and Meng, 1994; Woch and Lundin, 1992; Yamauchi et al., 1996). The cusps with their often complex magnetic topology and plasma structure relate to processes occurring elsewhere on the magnetopause (MP) and in the adjacent MSH in fashions which are at present not fully understood.
In this paper, we study a crossing of the high-latitude MP at the southern cusp, observed by Cluster on 13 February 2001. The general features of this event have been described by Cargill et al. (2004), who did not draw any definite conclusion on the nature of the MP crossing and by Dunlop et al. (2005), who showed evidence for an indentation region on the MP across the outer cusp and well defined plasma and magnetic boundaries, consistent with a funnel geometry. We further analyze this case in two companion papers. Here we present the observations, describe the MP crossing as a thin current sheet (TCS), located between the "incident" 
MSH plasma and a high speed jet, study the TCS movement, thickness and orientation, and show that proton finite gyroradius penetration through the TCS can account, at least in part, for the observed high speed jet; moreover, we briefly discuss the possible role of reconnection in this event. In the companion paper (Savin et al., 2006), cited further as "[S]", we discuss plasma acceleration, wave-particle interactions and Hall dynamics at the MP TCS.

\section{Overall description of the event}

We make use of data from four Cluster instruments: CIS, described by Rème et al. (2001), FGM, described by Balogh et al. (2001), PEACE, described by Johnstone et al. (1997), and EWF, described by Gustafsson et al. (2001). In the case under study, Cluster entered the magnetosphere through the southern cusp close to the GSM local noon. The Cluster orbit has been described in detail by Cargill et al. (2004) and Dunlop et al. (2005). Here we only recall that Cluster spacecraft 1 (SC1) was leading the fleet, closer to the Earth along the $\mathrm{x}$ GSM axis, while SC2, 3 and 4 were trailing in a plane roughly perpendicular to the $\mathrm{x}$-axis; the average distance between the spacecraft was $\sim 600 \mathrm{~km}$.

Figure 1 shows SC1 data in GSM from 19:15 to 20:15 UT on 13 February 2001. The vertical dashed line at 20:00:58 UT marks the MP crossing, for which Cargill et al. (2004) calculated a normal (in GSE) $N_{B}=(0.96,-0.21$, -0.16 ), averaged over the 4 spacecraft, and an outward speed of $30 \mathrm{~km} / \mathrm{s}$. In the following we break the description of the figure into individual paragraphs corresponding to the different panels.

Electric field. The top panel compares the $\mathrm{x}$ component of the electric field as calculated from EWF measurements under the hypothesis that $E \times B=0$ (black line) with $E_{x}$ (magenta line) as predicted by the Gas Dynamic Convection Field Model (GDCF). GDCF is a Spreiter code (e.g. see Song et al., 1999, and Savin et al., 2002a, c for details), for which we used as inputs interplanetary data provided by the SWEPAM and MAG instruments on board ACE at L1. A general agreement between the two quantities is observed, exception made for the 19:28-19:31 UT period and from 19:57 UT onwards. At 19:57 UT the model $E_{x}$ decreases, while the SC1 $E_{x}$ rises to about $6 \mathrm{mV} / \mathrm{m}$; then the model $E_{x}$ stays positive, while the SC1 $E_{x}$ goes through a minimum $\sim 0$ around 20:00 UT, displays a large bipolar oscillation roughly centered at the MP crossing and oscillates around 0 from 20:02 UT onwards. $E_{x}$ is a good proxy for the electric field along the quoted MP normal.

Clock angle. The second panel displays the magnetic field clock angle as calculated from SC1 data (black line) and through the GDCF model (magenta line). Until 19:57 UT a general agreement is observed, exception made for the 19:28-19:31 UT period, when they differ by $30-60^{\circ}$, and the 19:49-19:55 UT period, when a difference of the order of $20^{\circ}$ is observed. From 19:57 UT onwards the two clock angles totally disagree: the GDCF clock angle keeps close to $180^{\circ}$, while the $\mathrm{SC} 1$ angle first oscillates around $200^{\circ}$, then jumps by $\sim 70^{\circ}$ at 20:00:58 UT and oscillates around $140^{\circ}$ from 20:02 UT onwards.

Magnetic field components. The third panel shows the SC1 magnetic field components and the GDCF $B_{y}$. We notice that between 19:57 and 20:00 UT $B_{y}$ displays a dip through a $-30 \mathrm{nT}$ minimum around 19:58:30 UT which does not appear to be related to IMF changes; further on, at 20:00:58 UT the observed field turns towards its usual magnetospheric direction just inside the southern cusp: $\left|B_{z}\right|>B_{y}>\left|B_{x}\right|$, with $B_{z}<0$ and $B_{x} \sim 0$; this configuration is more clearly established at 20:08 UT.

Proton density and $B$. The fourth panel contains the total magnetic field intensity and the CIS-HIA proton number density multiplied by a 1.38 factor obtained by intercalibrating the CIS-HIA data with the WHISPER data (see Décréau et al., 2001). The total field oscillates around $60 \mathrm{nT}$ from 19:15 to 20:01 UT, when it drops to about $20 \mathrm{nT}$; after that, it oscillates at the new level for a few minutes and oscillates around $40 \mathrm{nT}$ from 20:03 to 20:08 UT, when it jumps to the normal magnetospheric value of $80 \mathrm{nT}$. The proton density varies between 10 and $25 \mathrm{~cm}^{-3}$ between 19:15 and 20:00 UT. At the MP itself it displays a small peak, which is hardly seen in Fig. 1, but will be further discussed in [S], followed by a decrease through a minimum of $9 \mathrm{~cm}^{-3}$ at 20:05 UT and a short lived maximum at 20:07 UT.

Proton temperature. The fifth panel shows the parallel and perpendicular proton temperatures: in the MSH generally $T_{\perp}>T_{\|}$; however, around 19:30 and 19:50 UT and from 19:55 to 20:00:58 UT $T_{\perp} \sim T_{\|}$; after the latter time, in correspondence with the dip of the total magnetic field, $T_{\perp}$ reaches its maximum and $T_{\perp}>T_{\|}$.

Proton velocity. The sixth and seventh panels display the proton velocity vector and the total and perpendicular speed respectively. The velocity is generally almost parallel to $B$, but is mainly perpendicular during four periods also characterized by enhanced flows: around 19:30 and 19:50 UT, from 19:55 to 19:59 UT and from 20:00:58 to 20:02 UT. By contrast, from 19:15 to 20:00 UT the GDCF velocity (not shown in the figure) is almost parallel to $B$, at an angle $<14^{\circ}$ on average. $V_{x}$ is generally of the order of $-50 \mathrm{~km} / \mathrm{s}$ and approaches 0 at 20:02 UT. Throughout the plot $V_{z}$ dominates as expected from the Cluster position, while $V_{y}$ is generally positive. Between 20:00:58 and 20:02 UT we observe a large jet, $230 \mathrm{~km} / \mathrm{s}$ on average and perpendicular to the magnetic field, mainly in $V_{z}$ and $V_{y}$; it is also worth noting that $V_{y}$ changes its sign at 20:00:58 UT from about 100 to $-70 \mathrm{~km} / \mathrm{s}$.

Alfvén speed and plasma beta. The eighth panel shows that the Alfvén Mach number is of the order of 0.5 most of the time, but increases to 1 over one minute prior to the MP crossing and reaches a maximum value of 3 in the jet. Finally, the proton $\beta_{\|}$and $\beta_{\perp}$ (last panel) are generally $\sim 1$, 


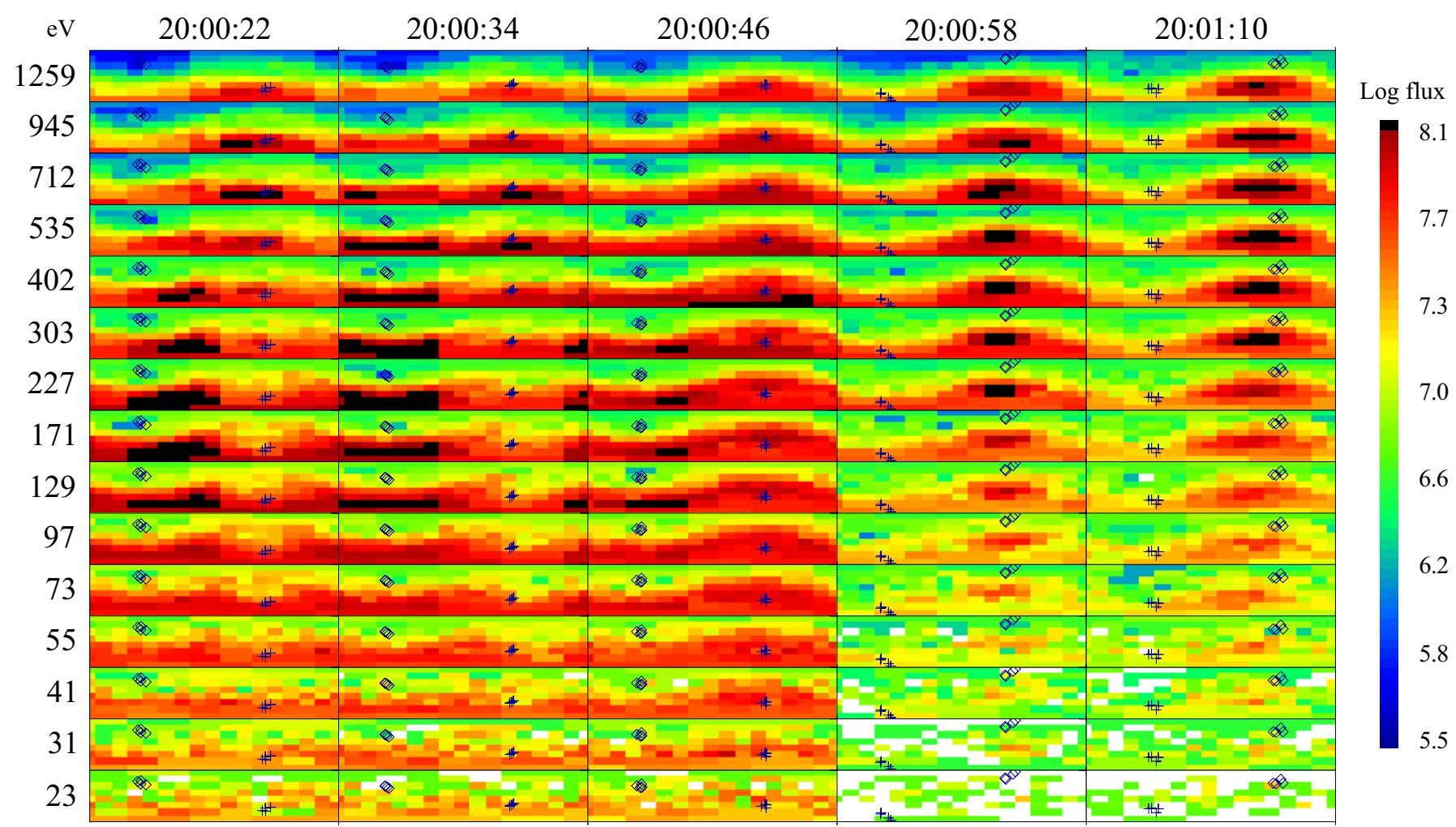

Fig. 2. Five successive CIS-HIA ion 3D flux distributions with $12 \mathrm{~s}$ resolution (starting times in UT at the top). For each acquisition period, 15 cells are piled up vertically, each corresponding to a given energy, lowest at the bottom $(23 \mathrm{eV})$, highest at the top (1259 eV). Each cell displays color-coded ion flux as a function, in the S/C system, of the azimuthal angle on the horizontal axis (from $0^{\circ}$ on the left to $360^{\circ}$ on the right) and the polar angle on the vertical axis (from $-90^{\circ}$ at the bottom to $90^{\circ}$ at the top). The magnetic field direction is shown in each cell by the crosses and rhombi which mark respectively the vector tip and end.

but are $>>1$ for several minutes after 20:00:58 UT, through a maximum value of 23 in the jet.

We conclude that the spacecraft is in the MSH until it crosses the MP at 20:00:58 UT. In the following section we demonstrate that ion flux crosses this boundary, which implies that the MP in this case cannot be described as a tangential discontinuity. The data show evidence that the MP is also approached around 19:30 and 19:50 UT and from 19:55 to 19:59 UT. To this regard, we recall that around 19:30, 19:50 and 20:00 UT 10-20 $\mathrm{keV} \mathrm{O}^{+}$(not shown) is observed, which can be considered as a proxy for the approach to the MP (e.g. see Phan et al., 2003). Between 20:00:58 UT and 20:06 UT the outer cusp is observed. Cargill et al. (2004) in their analysis of this event carried out a successful test for a deHoffmanTeller frame at the 20:00:58 UT boundary; they then tested the Walén relations and concluded that "consideration of all components gives negative slopes of approximately 0.8 ". It is well known that many authors regard this satisfactory for a rotational discontinuity (see e.g. Hultvist et al., 1999, and references therein). Accordingly, the interpretation of the observed MP transition as an RD could qualitatively account for the acceleration observed through it. However, Cargill et al. (2004) obtained, for the $\mathrm{x}, \mathrm{y}$ and $\mathrm{z}$ components, the fol- lowing slopes: $-0.54,-0.82,-0.6$ for Cluster 1 and -0.52 , $-1.12,-0.36$ for Cluster 3 . They concluded that "any identification of the MP as a rotational discontinuity is thus nondefinitive: the plasma flow is directed predominantly in the $y$ direction, perpendicular to the local magnetic field, no largescale density jump is seen, a decrease in the field magnitude and a rise in temperature are observed, the magnetic field rotates by $90^{\circ}$ to point predominantly in the Z-direction. A variety of MHD boundaries satisfies some of these conditions, but none satisfies them all (see also Lavraud et al., 2002)." On these grounds, in this paper and in [S] we propose an alternate explanation for the acceleration observed in this case at the MP.

\section{Detailed description of the MP transition}

Figure 2 shows five successive CIS-HIA ion 3D flux distributions with $12 \mathrm{~s}$ resolution between 20:00:22 and 20:01:22 UT. The 20:00:58 UT MP crossing occurs just at the beginning of the fourth acquisition. For each acquisition period, 15 cells are plotted vertically, each corresponding to a given energy, lowest at the bottom $(23 \mathrm{eV})$, highest at the top $(1259 \mathrm{eV})$. 


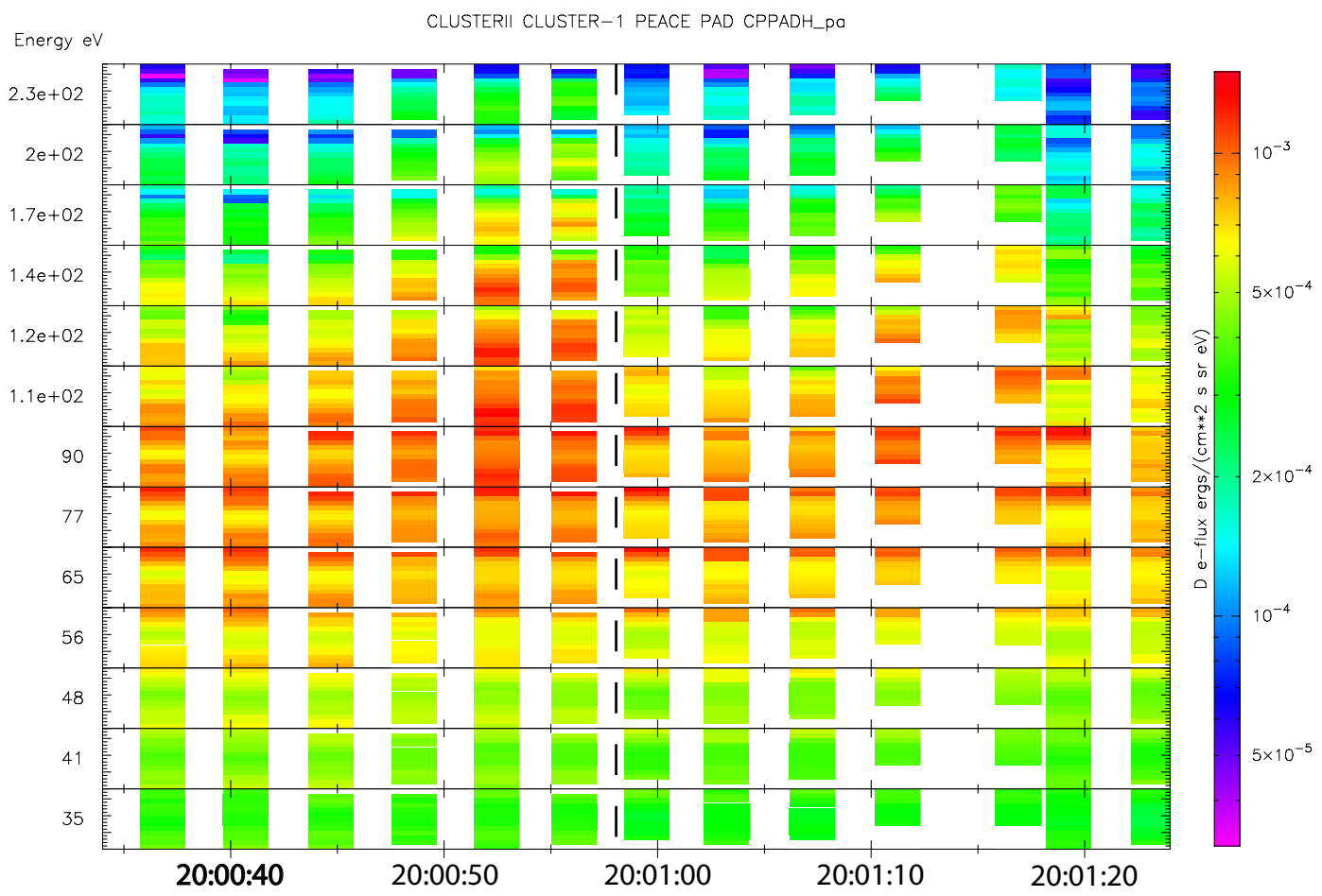

Fig. 3. Pitch angle distribution of colour coded differential energy flux from the PEACE high energy detector (HEEA) between 20:00:34 and 20:01:22 UT for 13 energy channels from 33 to $250 \mathrm{eV}$. For each distribution the pitch angle on the vertical axis varies between $0^{\circ}$ (bottom) and $180^{\circ}$ (top). The MP at 20:00:58 UT is marked by a vertical dashed line.

Each cell displays color-coded ion flux as a function, in the non-rotating S/C system, of the azimuthal angle (from $0^{\circ}$ to $360^{\circ}$ on the horizontal axis) and the polar angle (from $-90^{\circ}$ to $90^{\circ}$ on the vertical axis). In each cell crosses and rhombi mark tips and ends of magnetic field vectors respectively. As shown already in Fig. 1, the magnetic field clock angle abruptly rotates by $\sim 70^{\circ}$ at the MP. Prior to the MP crossing the protons display two populations which partly overlap in energy but have different directions, roughly parallel and perpendicular to $B$ respectively. Careful inspection over the preceding 20 min of similar plots of CIS-HIA distributions (not shown herein) reveals that the roughly perpendicular population is continuously observed and that the parallel one is present for about $5 \mathrm{~min}$ prior to the MP crossing. Therefore, we interpret the first one as the main MSH plasma population, while the nearly parallel one resembles the "reflected" population often observed in the MSH boundary layer (e.g. see Fuselier et al., 1997). In the first distribution on the left the perpendicular population peaks around $120^{\circ}$ in azimuth and around $300 \mathrm{eV}$ in energy; by contrast in the top 5 energy channels the parallel one shows up around the magnetic field vector tip, i.e. at $260^{\circ}$ in azimuth and $-40^{\circ}$ in polar angle. In such angular bins the lower energy channels display a lower flux, to which both populations probably contribute. In the second distribution the perpendicular population is somewhat enhanced. The third distribution from the left is the last before the MP crossing and shows already a definite change, so that the two populations are harder to distinguish. Nevertheless, we remark three flux peaks in the middle energy channels, around $130^{\circ}$ in azimuth, roughly perpendicular to $B$, while the main distribution is now around the magnetic field, extending from low to high energy with a peak around $400 \mathrm{eV}$. After the MP crossing at 20:00:58 UT, when, as described in Fig. 1, the speed enhancement starts, a single population is observed, roughly perpendicular to the local magnetic field, but at azimuthal and polar angles similar to those of the parallel population in the MSH and energies somewhat higher, peaked around $700 \mathrm{eV}$, and with higher fluxes. We also notice that, for all angular bins, the flux at energies below $100 \mathrm{eV}$ is much lower than before the MP crossing. Careful inspection of similar plots for the successive CISHIA distributions shows that the jet population is continuously observed for about $60 \mathrm{~s}$ after the MP crossing.

Figure 3 shows the pitch angle distribution of colour coded differential energy flux from the PEACE high energy detector (HEEA) between 20:00:34 and 20:01:22 UT for 13 energy channels from 33 to $250 \mathrm{eV}$. The MP at 20:00:58 UT is marked by a vertical dashed line. For each distribution the pitch angle on the vertical axis varies between $0^{\circ}$ (bottom) and $180^{\circ}$ (top). The five lowest energy channels show bi-directional unbalanced electrons, flowing mainly anti-parallel, while electrons above $100 \mathrm{eV}$ flow mainly par- 


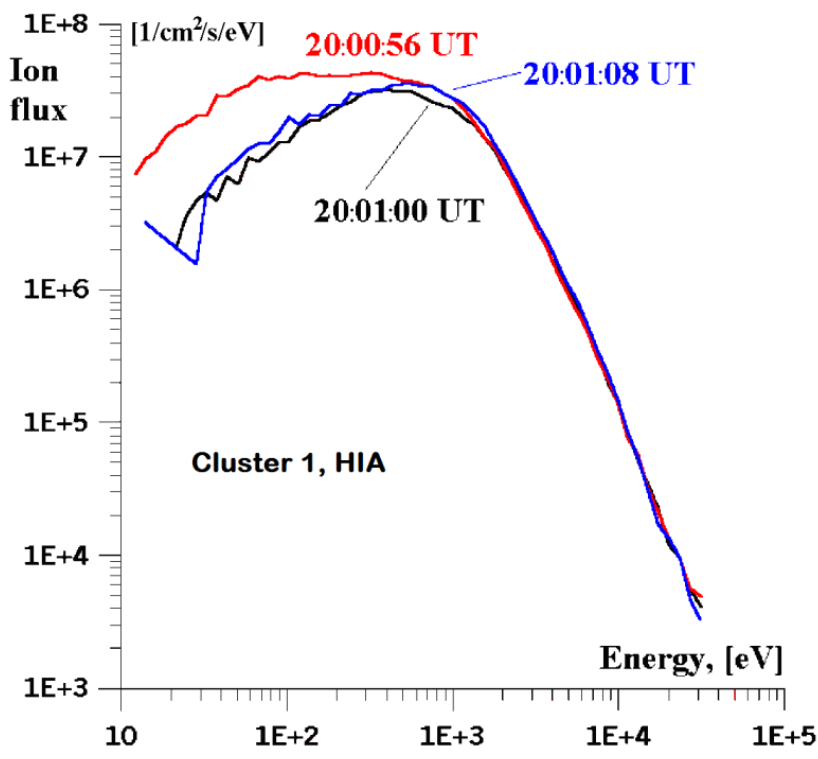

Fig. 4. $4 \mathrm{~s}$ resolution CIS-HIA omni-directional differential proton fluxes as a function of energy, pertaining to three S/C spins around the MP crossing (central times are given in UT).

allel to the magnetic field both in the MSH and across the MP until 20:01:10 UT. As the IMF is southward oriented, this can be interpreted as due to dayside reconnection of the magnetic field line threading through the Cluster position. Intensification of the higher-energy parallel flux just prior to the MP in conjunction with the velocity rise (see Fig. 1) is another feature which fits with dayside reconnection as the source of the heated electrons. However, it is not clear why perpendicular fluxes are substantially lower than parallel and anti-parallel fluxes at the lower energies. Another feature, unexplained by a remote source, is the presence of intense quasi-perpendicular electrons at higher energies $77-230 \mathrm{eV}$ just prior to the MP.

In Fig. 4 we display, as a function of energy, $4 \mathrm{~s}$ resolution omni-directional differential proton fluxes from CISHIA pertaining to three spacecraft rotations around the MP crossing. The use of omni-directional distributions is justified in this case by the fact that the parallel and perpendicular populations in the MSH and the jet population have already been clearly identified. The red line shows proton fluxes measured just prior to the MP crossing, between 20:00:54 and 20:00:58 UT: a broad maximum extends from $60 \mathrm{eV}$ to $500 \mathrm{eV}$, which we interpret as due to the contributions of the parallel and perpendicular populations in the MSH. Inspection of the CIS-HIA on board calculated moments shows that the proton velocity at this time is $\sim 175 \mathrm{~km} / \mathrm{s}$, mainly parallel to $B$. The black line shows proton fluxes measured at the MP and immediately downstream (central acquisition time is 20:01:00 UT): the spectrum is peaked at $400 \mathrm{eV}$ and its high energy tail coincides roughly with that of the previous one; on the other hand, at lower energies the flux is greatly reduced (by one order of magnitude at $20 \mathrm{eV}$ ). At this time the on board calculated velocity is perpendicular to $B$ and $\sim 240 \mathrm{~km} / \mathrm{s}$. The blue line shows proton fluxes further tailward (central acquisition time is 20:01:08 UT): the high energy tail of the distribution coincides with that of the previous one, while the peak now occurs around $500 \mathrm{eV}$. These observations confirm that the change of the 3D distributions described in Fig. 2 occurs just at the MP crossing on a time scale probably shorter that the spacecraft $4 \mathrm{~s}$ spin period. We can give a crude estimate of the MP thickness by multiplying the $30 \mathrm{~km} / \mathrm{s}$ MP speed quoted by Cargill et al. (2004) by $2-4 \mathrm{~s}$; this yields $60-120 \mathrm{~km}$. On the other hand, the magnetic field intensity during the MP crossing is $\sim 30 \mathrm{nT}$, which yields for $0.2-1.0 \mathrm{keV}$ protons a Larmour radius $\rho \sim 68-153 \mathrm{~km}$. These qualitative calculations and the observations we have described in Figs. 2 and 4 lead us to propose that lower energy protons could be stopped by the MP TCS, while higher energy ones could move freely across it.

Starting from this hypothesis, we recall that the dominant electric field component across the MP is $E_{x}$, which lies mostly along the MP normal as calculated by Cargill et al. (2004). $E_{x}$ drops by $5 \mathrm{mV} / \mathrm{m}$ over just $2 \mathrm{~s}$, between 20:00:58 and 20:01:00 UT (as shown in Fig. 1 and, in more detail, in Fig. 8), i.e. over $60 \mathrm{~km}$, given the MP speed of $30 \mathrm{~km}$. The integration of $E_{x}$ across such a distance yields a potential jump of $\sim 300 \mathrm{~V}$ (cf. Vaivads et al., 2004), suggesting that protons crossing the MP could be accelerated and gain an energy of the order of $300 \mathrm{eV}$. To check this, at least semi-quantitatively, we extract, from the $12 \mathrm{~s}$ resolution 3-D CIS-HIA distributions, proton fluxes summed over $45^{\circ}$ in azimuth and polar angle in the tailward direction. Figure 5 shows such fluxes for two successive distributions, centered respectively at 20:00:52 UT (black curve), just prior to the MP, and 20:01:04 UT (blue curve), just after it. The two spectra cross each other around $300 \mathrm{eV}$, being the black curve higher at lower energies and lower at higher energies. If we make the hypothesis that the higher energy MSH protons penetrate through the thin current sheet thanks to their large gyroradius and are energized by $E_{x}$, we should obtain the fluxes on the MP cusp side (blue curve) by appropriately shifting in energy the MSH fluxes. In fact, between 1 and $4 \mathrm{keV}$ the blue curve roughly coincides with the red curve, which is obtained by shifting to the right the higher energy part of the black curve (MSH fluxes) by $200 \mathrm{eV}$. Similarly, at energies higher than $5 \mathrm{keV}$ the blue curve roughly coincides with the cyan curve, obtained by shifting the higher energy part of the black curve by $300 \mathrm{eV}$. This suggests that indeed larger gyroradius protons are able to penetrate through the MP roughly parallel to $E_{x}$ and are energy shifted by the cross-MP potential.

As we are considering the fine structure of the MP sheet, it is natural to make also use of CIS-HIA data from SC3 (while such data are not available for SC2 and 4), which is trailing $\mathrm{SC} 1$ at $600 \mathrm{~km}$ distance. The MP was crossed by SC3 


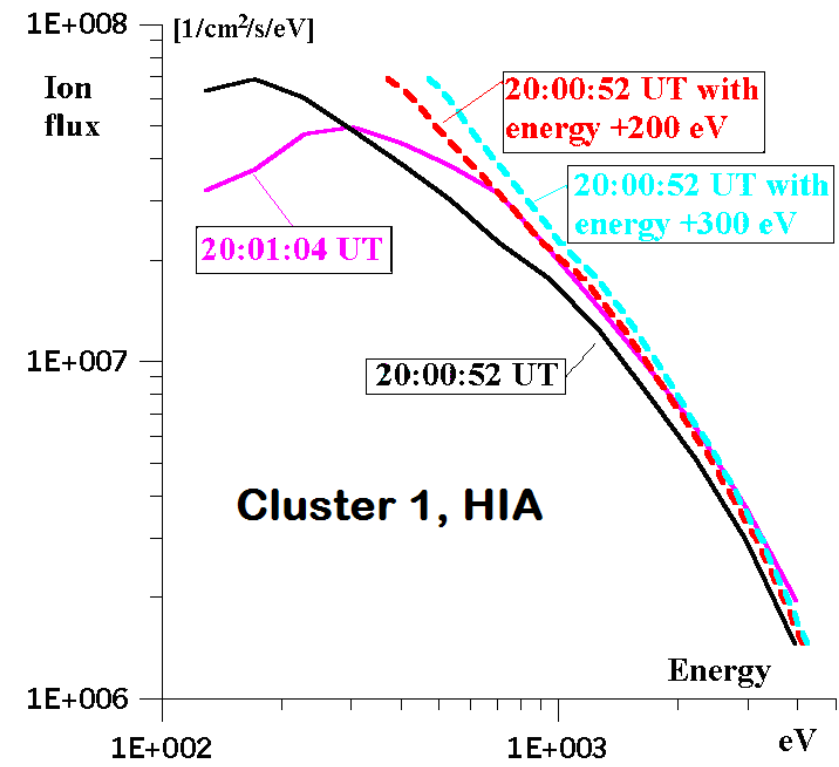

Fig. 5. Same format as in Fig. 4a, for fluxes summed over $45^{\circ}$ in azimuth and polar angle in the tailward direction from 2 full $3 \mathrm{D}$ distributions (12 $\mathrm{s}$ resolution).

slightly later than by SC1 (cf. Cargill et al., 2004) and careful examination of the data (see Fig. 8 below) suggests that this occurred around 20:01:02 UT. Figure 6 displays four subsequent omni-directional spectra in the same format as in Fig. 4 ( $4 \mathrm{~s}$ resolution). As for Fig. 4, we have carefully compared such omni-directional spectra with the corresponding $12 \mathrm{~s}$ 3-D spectra (not shown herein). The spectrum centered at 20:00:58 UT (black line) pertains to the MSH and shows a lower energy and a higher energy peak, which we interpret as due to the two distinct MSH populations described in Fig. 2. The spectrum centered at 20:01:02 (blue line) is measured just around the MP crossing and displays a slight depletion at lower energies. The violet and red lines are measured inside the high speed jet, centered at 20:01:06 and 20:01:10 UT respectively, and clearly show a depletion at lower energies, as already seen for $\mathrm{SC} 1$ in Fig. 4. Again we propose, as for $\mathrm{SC}$, that larger gyroradius protons can move across the boundary, while smaller gyroradius protons are confined in the MSH boundary layer.

Figure 7 displays four SC1 4 s omni-directional spectra: the last MSH spectrum, red line (centered at 20:00:56 UT), is shown for reference. The black and green lines refer to spectra centered at 20:01:00 and at 20:02:33 UT respectively, the first one, peaked at $500 \mathrm{eV}$, on the MP side of the speed jet, the second one, peaked at $100 \mathrm{eV}$, on the opposite side, $30 \mathrm{~s}$ after the end of the speed jet. The spectra between 20:01:00 and 20:02:33 UT (not shown in the figure in order not to overload it) display a gradual shift to lower energies. Finally, the mantle is here represented by the thin blue line spectrum, centered at 20:08:27 UT, which displays a dense and cold

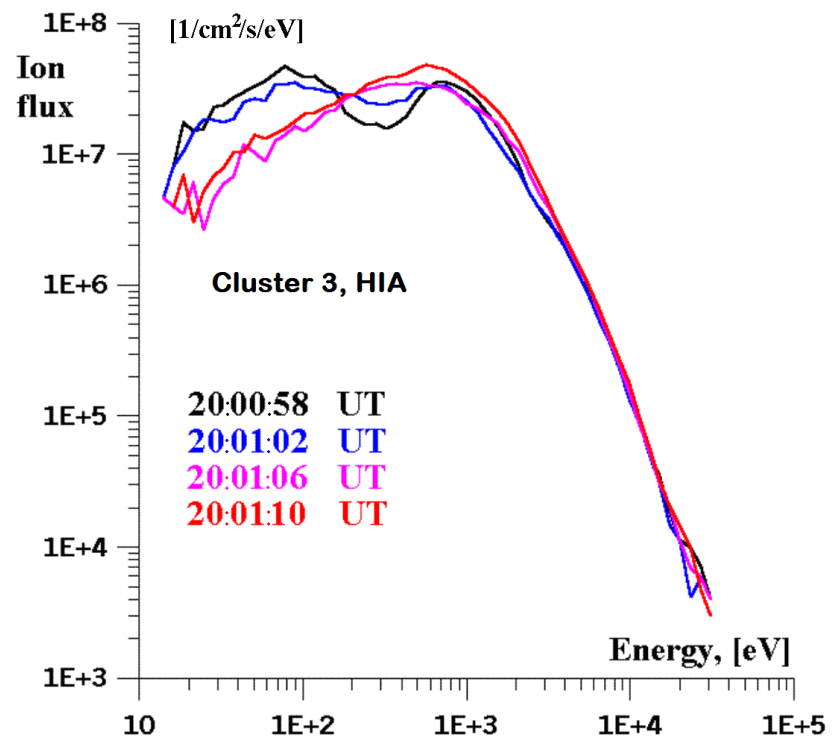

Fig. 6. Four SC3 4 s resolution omni-directional spectra in the same format as in Fig. 4 around the TCS crossing.

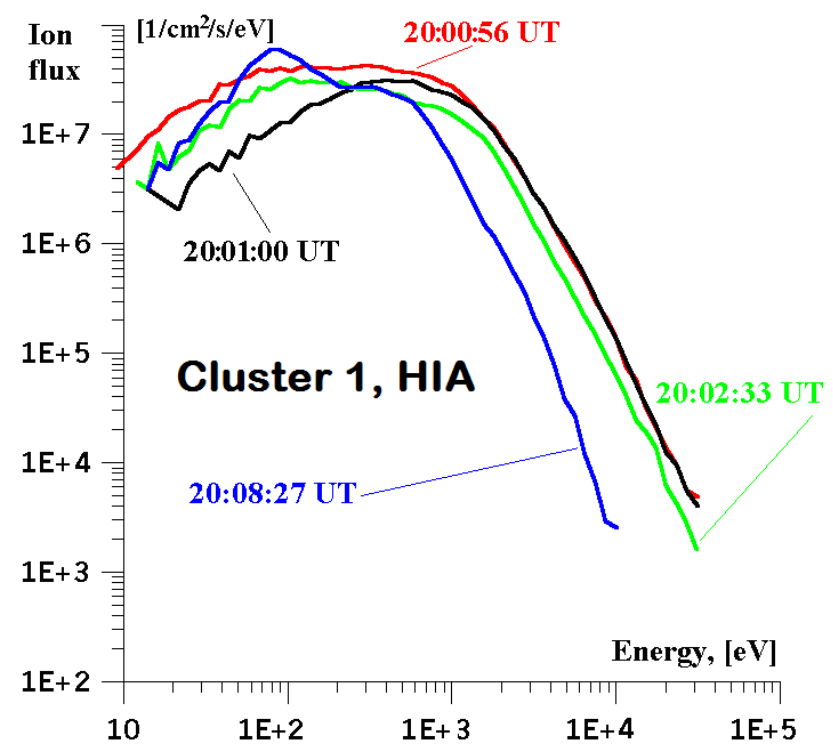

Fig. 7. SC1 4 s omni-directional spectra at four different times (same format as in Fig. 4).

low energy mantle population, peaked just below $100 \mathrm{eV}$ and a higher energy shoulder above $200 \mathrm{eV}$. To this regard, the inspection of $12 \mathrm{~s} 3$-D distributions reveals that the lower energy 'mantle' population is parallel to the magnetic field and the higher energy population is mainly perpendicular, resembling the shape of the spectra in the 20:01:04-20:02:33 UT period but with much lower density.

To conclude the description of the MP crossing we compare the cross-field drift velocity $V_{d}=E \times B$ to the proton velocity perpendicular to $B$. Figure 8 displays the three GSE 


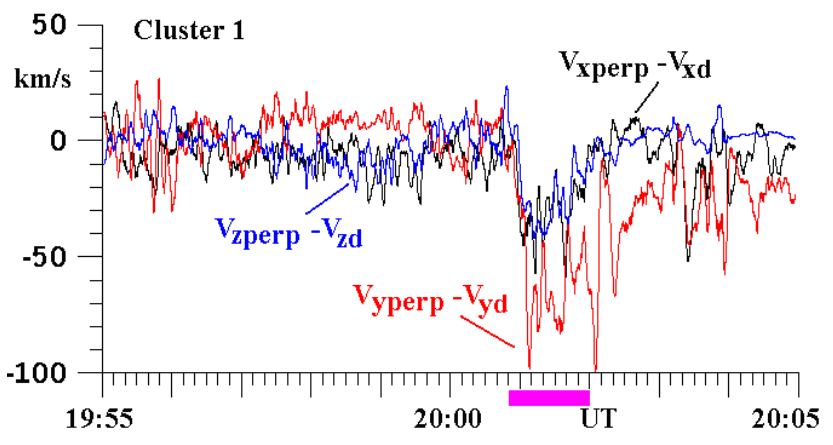

Fig. 8. Three components of the difference in $[\mathrm{km} / \mathrm{s}]$ between the ion perpendicular velocity and electric drift velocity $V_{\perp}-V_{d}$ in GSE.

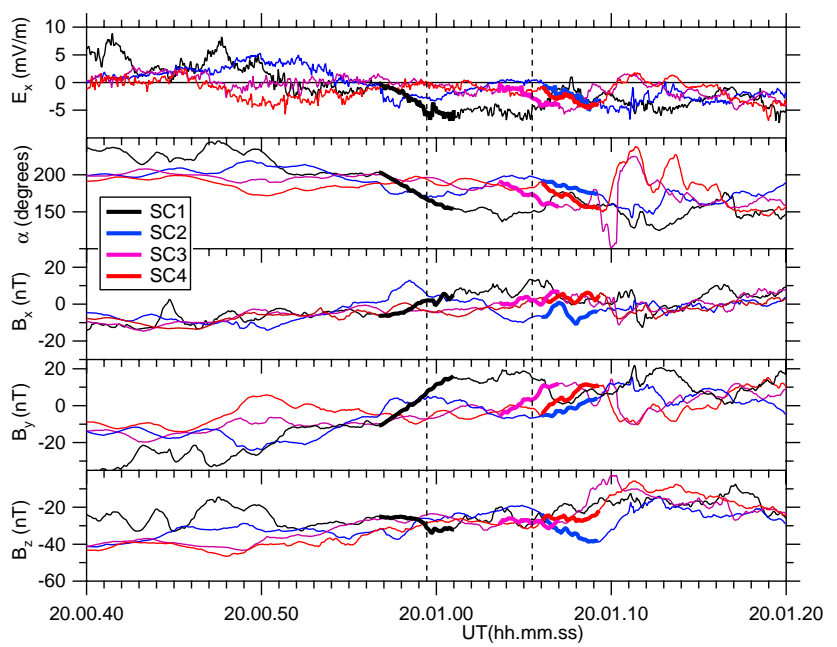

Fig. 9. From top to bottom: $E_{x}$, magnetic field clock-angle, and $B_{x}, B_{y}, B_{z}$. In each panel data from SC1-4 are presented in GSE. The two vertical dashed lines mark the MP crossing at SC1 (black) and SC3 (magenta). In each panel the thicker curves indicate the TCS crossings for each spacecraft (see text for details).

components of $V_{\text {perp }}-V_{d}$ for SC1 from 19:55 to 20:05 UT with $4 \mathrm{~s}$ resolution, where $V_{\text {perp }}=V_{o b}-\left(V_{o b} \cdot B\right) B / B$ and $V_{o b}$ is the on board calculated proton velocity. From 19:55 to 20:00 UT the three traces keep close to 0 , implying that $V_{\text {perp }}$ and $V_{d}$ practically coincide. This occurs in spite of a non-equilibrium plasma with two co-existing ion distributions with different moments, as shown in Fig. 2, which should not necessarily follow a single-fluid Ohm equation (cfr. Sibeck et al., 1999). Between 20:01 and 20:02 UT all three components depart significantly from 0 . In particular, this is true for the y component, which deviates from 0 also in the 20:02-20:05 UT period. The average of $V_{y \text { perp }}-V_{d y}$ over the interval 19:55-20:02 UT is $\sim 2 \mathrm{~km} / \mathrm{s}$, while the corresponding standard deviation is $15 \mathrm{~km} / \mathrm{s}$. Inside the magnetosphere, between 20:04 and 20:05 UT, the average $V_{y \text { perp- }}$ $V_{d y}$ is $\sim-20 \mathrm{~km} / \mathrm{s}$ (with standard deviation $14 \mathrm{~km} / \mathrm{s}$ ). To be cautious, one can attribute such two different average values to different offsets in the $E_{x}$ component of EFW in the MSH and in the more dilute magnetospheric plasma (anyway, the offset difference appears to be much smaller for the other electric field components). The average of $V_{y \text { perp }}-V_{d y}$ over the 20:01-20:02 UT speed jet (marked by the violet bar at the right bottom side of Fig. 8) is $\sim 64 \mathrm{~km} / \mathrm{s}$, with standard deviation $45 \mathrm{~km} / \mathrm{s}$, amounting to $29 \%$ of the average velocity measured in the jet. If we attribute to the jet the same $E_{x}$ offset as in the 20:02-20:05 UT period, this would reduce the average deviation $V_{y \text { perp }}-V_{d y}$ to $44 \mathrm{~km} / \mathrm{s}$, i.e. to $20 \%$ of the total speed. However, between 20:01 and 20:02 UT the offset in the EFW $E_{x}$ measurement is probably closer to the MSH one, as in the jet the density is half way between the MSH value and the value after the jet. In conclusion, we take as a fair and reasonable estimate for $\left|V_{\text {perp }}-V_{d}\right|$ in the jet a value of $57 \mathrm{~km} / \mathrm{s}$, i.e. $25 \%$ of the total speed. The discussion of this difference in terms of local input due to ion finite gyroradius penetration in further pursued in $[\mathrm{S}]$.

\section{Magnetopause TCS width, orientation and motion}

It is known that the motion of the cusp boundaries can occur at speeds up to $60 \mathrm{~km} / \mathrm{s}$ and be due to global motion of the cusp, to its expansion or to its deflation. For the cusp entry on 13 February 2001, close inspection of the full magnetic shear reveals that the spacecraft crossing order does not imply a simple outward motion of the boundaries across the spacecraft fleet (cf. Cargill et al., 2004). Therefore, in this paragraph we consider in more detail the motion, normal and thickness of the MP layer.

First of all it must be remarked that such quantities can be determined on various spatial and temporal scales. The normal and speed quoted by Cargill et al. (2004) refer to $2 \mathrm{~min}$ utes around the MP crossing, from 19:59:30 to 20:01:30 UT. Here we will consider both somewhat longer time periods, 4 and $3 \mathrm{~min}$, and much smaller ones, of the order of a few seconds.

We first determined the large scale MP velocity from $E_{x}$ data. For this purpose we used a four minute period around the MP crossing, from 19:59:00 to 20:03:00 UT. We recall that $\mathrm{SC} 2,3$ and 4 lie in a plane almost parallel to the MP plane, while SC1 is leading the fleet by about $600 \mathrm{~km}$ and is the first to cross the MP. Cross correlation of the $\mathrm{E}_{x}$ data from the four spacecraft shows that the $E_{x}$ waveforms for $\mathrm{SC} 2,3$ and 4 have small lags and high correlation coefficients, around $93 \%$, while the SC1 waveform looks clearly shifted in time with respect to the other three. The best crosscorrelation coefficient between the signals on $\mathrm{SC} 1$ and 3 over the same 4-min interval is found to be 0.8 for a time lag of $18 \mathrm{~s}$. Under the assumption of MP planarity and time stationarity, the three lags between the Cluster spacecraft yield a MP velocity $V_{\mathrm{MP}}=(26.2 ; 0.2 ;-15.7) \mathrm{km} / \mathrm{s}$ in GSE, using the constant velocity approach (CVA, see Haaland et al., 2004). We 
calculated the MP normal at the four S/C from $B$ minimum variance analysis over the 19:58:47-20:02:07 UT period and got results within $7^{\circ}$ from each other. As an example, $N_{B 3} \sim$ $(0.97 ;-0.19 ;-0.15)$, i.e. close to the Cargill et al. (2004) normal and at $\sim 25^{\circ}$ from $V_{\text {MP. These }} 25^{\circ}$ might be regarded as an estimate for the validity of the MP coplanarity and stationarity hypotheses, but they can also have a specific physical meaning (we will return to this point in [S]).

We now turn to smaller scales. Figure 9 displays EFW and FGM data (in GSE) from the 4 Cluster spacecraft for a closeup of the MP crossing between 20:00:40 and 20:01:20 UT (SC1-4 data are shown in black, blue, violet and red respectively). From top to bottom we have: $E_{x}$, magnetic clockangle, $B_{x}, B_{y}, B_{z}$. Two vertical dashed lines indicate the MP crossings by SC1 and SC3 (black and magenta lines respectively). As a starting point for our analysis we consider the SC1 clock angle and notice that around the MP crossing it rotates by about $40^{\circ}$ over $3 \mathrm{~s}$; subsequently, we identify the corresponding rotations for the other three spacecraft and evidence them all as thicker portions of the 4 clock angle curves. Correspondingly we draw thick portions for the curves pertaining to each field component and spacecraft. We remark that the TCS identified for SC1 and SC3 correspond to the plasma separatrices in Figs. 2 and 5.

We tried to perform a minimum variance analysis of the magnetic field data for each spacecraft for the small interval we have just described. Such analysis does not produce acceptable results. The reason can be found through a careful inspection of the various $B$ components, which shows that, while at the TCS all four $B_{y}$ 's change consistently sign from negative to positive, the behavior of $B_{x}$ and $B_{z}$ is rather irregular. Having discarded the $B$ minimum variance, we used the maximum variance analysis for the electric field over 5$6 \mathrm{~s}$ around the MP crossings and obtained the following GSE normals (eigen values in brackets):

SC1: $0.9,-0.43,0.07(0.0025,0.065,5.26)$;

SC2: $0.95,-0.3,0.087(0.0026,0.469,4.2)$;

SC3: $0.967,-0.2,0.158(0.0024,0.138,2.44)$;

SC4: $0.964,-0.23,0.133(0.0025,0.32,1.31)$.

On this basis, we accepted as common normal $N_{E}=(0.95$, $-0.3,0.087)$, which is at $16^{\circ}$ to the averaged large-scale normal from Cargill et al. (2004) and at $40^{\circ}$ to the average largescale velocity $V_{\text {MP }}$ (see discussion above). We then calculated the MP velocity by two different methods. Firstly, we used again the CVA, this time on a $20 \mathrm{~s}$ time period, comprising of the four MP crossings, and obtained $V_{C V A} \sim 74^{*}(0.78$, $-0.43,-0.46) \mathrm{km} / \mathrm{s}$. This MP velocity does not agree at all with $V_{\mathrm{MP}}=(26.2 ; 0.2 ;-15.7) \mathrm{km} / \mathrm{s}$ calculated from $E_{x}$ crosscorrelation and yields a MP thickness at SC1 $d_{\mathrm{CVA}} \sim 166 \mathrm{~km}$ along $N_{E}$. Dunlop et al. (2004) also argue that the "timing" normals (i.e. CVA ones) are affected by complicated MP accelerations. Then we used the CTA (Constant Thickness Approach; see Haaland et al, 2004) and obtained a MP width $d_{\mathrm{CTA}} \sim 90 \mathrm{~km}$, a normal $N_{\mathrm{CTA}} \sim(0.815,0.579,0.02)$ and four speeds $V_{\mathrm{CTA}} \sim 41,35,43,33 \mathrm{~km} / \mathrm{s}$, for $\mathrm{SC} 1$ through 4 respectively. This yields that the $\mathrm{x}$ component of the MP velocity at $\mathrm{SC} 1$ be $\sim 32 \mathrm{~km} / \mathrm{s}$.

These values strongly support the analysis made in Sect. 3: firstly, $90 \mathrm{~km}$ is the gyroradius of a $\sim 350 \mathrm{eV}$ proton in a $30 \mathrm{nT}$ magnetic field, which fairly well agrees with the low-energy cutoff observed in Figs. 4 and 6 across the TCS; in the second place, the large-scale MP velocity value $\left(\left|V_{\mathrm{MP}}\right| \sim 30 \mathrm{~km} / \mathrm{s}\right)$ lies in a $21 \%$ window from the averaged CTA one $(38 \mathrm{~km} / \mathrm{s})$, that is an acceptable agreement between MP velocities calculated over different spatial scales. As a conclusion of this analysis, we take $90 \mathrm{~km}$ as the width of the TCS, $x$ GSE as a good proxy for its normal and $30-35 \mathrm{~km} / \mathrm{s}$ as the $\mathrm{x}$ component of its velocity, directed towards the Sun. We will return to the differences in the normal directions in $[\mathrm{S}]$.

\section{Summary and conclusions}

We summarize and discuss our findings on a kinetic effect, namely the penetration of ions through a TCS by finitegyroradius, to explain plasma and field observations made by Cluster at a MP crossing in the southern cusp around 20:01 UT on 13 February 2001.

First of all, we wish to briefly discuss the possible role of reconnection in this event. The orientation of the MSH magnetic field in the one hour period displayed in Fig. 1 is such that reconnection is expected to occur at the dayside magnetopause. The PEACE electron data shown and discussed in Fig. 3 suggest that the magnetic field lines crossed by Cluster around the MP TCS be probably connected to such a dayside reconnection site, although the same electron data show unexpected features. We tend to exclude that Cluster is close to a reconnection diffusion region. We realize that a common way of interpreting the crossing of a MP in such a situation would be to postulate the local change of bulk velocity according to the Walén relation. However, as recalled earlier, the Walén test in this case is not fully satisfactory, as already established by Cargill et al. (2004). For this reason, we proceeded to further analyze the TCS on a gyroradius space scale.

We compared the Cluster observations with the predictions of the Gas Dynamic Convection Field (GDCF) model in the $\mathrm{MSH}$ and found a general good agreement between the two. However, the observations depart from the model starting from the MSH boundary layer. In particular, we found that, close to the MP, on its inbound orbit, Cluster measured an anti-sunward electric field opposite to the GDCF predictions and that the Cluster clock-angle rotation also mismatched the GDCF predictions. The main electric field component on both sides of the MP is found to lie along x GSE and displays a bipolar signature, positive on the MSH side and negative on the opposite side. As it will be further discussed in [S], we interpret this field as due to a positive surface charge at the MP, 
which moves outward at $30-35 \mathrm{~km} / \mathrm{s}$. Further on, the careful examination of 3-D proton spectra and of the field data suggested us the existence of a Thin Current Sheet (TCS) at the MP, observed by SC1 between 20:00:57 and 20:01:01 UT. Starting from the conclusion by Cargill et al. (2004), who ruled out that such discontinuity is tangential in nature, but could not determine whether it can be classified as rotational, we further studied the TCS and concluded that it has a width of $90 \mathrm{~km}$, a normal mainly directed along x GSE and a speed of $30-35 \mathrm{~km} / \mathrm{s}$ towards the incoming MSH plasma.

3-D distributions of protons have been carefully examined for SC1 between 19:50 and 20:10 UT, around the 20:00:58 MP crossing. Two populations have been identified in the MSH boundary layer: the first one roughly perpendicular to the MSH magnetic field, which we interpret as the "incident" MSH plasma, the second one mostly parallel to $B$. We do not make specific hypotheses on the origin of the second population, but recall that many authors regard this, in the presence of reconnection, as a "reflected" population (see e.g. Fuselier et al., 1997). In the last distribution just before the MP crossing, between 20:00:46 and 20:00:58 UT, the main distribution is observed around the magnetic field, extending from low to high energy, while the one roughly perpendicular to $B$ is still seen with a peak around $400 \mathrm{eV}$. Correspondingly, just in front of the MP the Alfvén Mach number increases from 0.8 to 1.0 and $\beta$ rises from 1 to 5 . After the MP crossing a single population is observed, roughly perpendicular to the local magnetic field, which results in a large speed jet, where $V \sim 230 \mathrm{~km} / \mathrm{s}$ on average, the Alfvén Mach number goes through a maximum of 3 , and $\beta$ oscillates around 10 with a peak value of 23 . The $E \times B$ velocity just before the MP coincides with the perpendicular velocity measured by CIS, implying that the local electric field is responsible for deflecting the flow of the MSH plasma along the MP. Instead, in the speed jet a difference between the two is observed, amounting to about $25 \%$ of the total speed. We suggest that the measured $E$ can account for the main plasma flow, but the jet can be, at least partly, interpreted in terms of finite gyroradius penetration through the TCS (see [S] for further details on that).

We further studied the transition around the TCS by considering omni-directional proton fluxes in order to increase the time resolution to $4 \mathrm{~s}$. For that purpose we used both SC1 and SC3 data and showed that they made comparable observations, suggesting that the high energy parts of the distributions appear to largely coincide on the two sides of the TCS, while the low energy portion appears to be greatly reduced across it from the MSH to the cusp side. We propose that the experimental data can be explained by the hypothesis that the TCS acts locally as an obstacle for low-energy ions $(<350 \mathrm{eV})$, being transparent for ions with larger gyroradius. As in the jet $\beta \sim 10$, ions crossing the MP due to the finite-gyroradius effect should nearly conserve their momentum parallel to the MP surface. In fact, we observed that in the last $12 \mathrm{~s} 3-\mathrm{D}$ distribution and in the corresponding
$4 \mathrm{~s}$ omni-directional distributions measured before the MP crossing the parallel flow dominates (see Figs. 1, 2, 4), while the flow becomes perpendicular downstream of the MP and the low energy component both parallel and perpendicular to $B$ is greatly reduced. As $\beta>>1$, the plasma dominates the flow, so that downstream of the MP $B$ adjusts to it and to $E$ through a nearly $90^{\circ}$ rotation. We further presented evidence on a $12 \mathrm{~s}$ time scale that protons which cross the TCS by finite gyroradius penetration are locally energized by the $E_{x}$ field by $200-300 \mathrm{eV}$. This does not take place for the lower energy protons which do not cross the TCS because of their small gyroradius.

The electrodynamics of the TCS, mass and momentum transfer across it and the probable spatial extension of the jet are further discussed in [S]. In that paper further considerations are also made on the role of dayside reconnection.

Acknowledgements. This work was partially supported by INTAS03-50-4872, INTAS 05-1000008-8050, RFFS 06-02-17256, ISSI (Team 81), KBN 8T12 E 016 28, and ECRT Network HPRN-CT2001-0314.

Edited by: D. Vassiliadis

Reviewed by: one referee

\section{References}

André, M., Vaivads, A., Buchert, S. C., Fazakerley, A. N., and Lahiff, A.: Thin electron-scale layers at the magnetopause, Geophys. Res. Lett., 31, L03803, doi:10.1029/2003GL018137, 2004

Balogh, A., Carr, C. M., Acuña, M. H., Dunlop, M. W., Beek, et al.: The Cluster magnetic field investigation: overview of in-flight performance and initial results, Ann. Geophys., 19, 1207-1217, 2001 , http://www.ann-geophys.net/19/1207/2001/.

Belmont, G. and Rezeau, L.: Magnetopause reconnection induced by Hall-MHD fluctuations, J. Geophys. Res., 106(A6), $10751-$ 10760, 2001.

Blecki, J., Rothkael, H., Kossacki, K., Wronowski, R., Klos, Z., Juchnievicz, J., Savin, S., et al.: ULF-ELF-VLF-HF plasma wave observations in the polar cusp onboard high and low altitude satellites, Physica Scripta, 75, 259-263, 1998.

Boardsen, S. A., Eastman, T. E., Sotirelis, T., and Green, J. L.: An empirical model of the high-latitude magnetopause, J. Geophys. Res., 105, 23 193-23 220, 2000.

Cargill, P. J., Dunlop, M. W., Lavraud, B., Elphic, R. C., Holland, D. L., et al.: CLUSTER encounters with the high altitude cusp: boundary structure and magnetic field depletions, Ann. Geophys., 22, 1739-1754, 2004,

http://www.ann-geophys.net/22/1739/2004/.

Chen, S.-H., Boardsen, S. A., Fung, S. F., Green, J. L., Kessel, R. L., Tan, L. C., Eastman, T. E. and Craven, J. D.: Exterior and interior polar cusps: Observations from Hawkeye, J. Geophys. Res., 102(A6), 11335-11348, 1997.

Décréau, P. M. E., Fergeau, P., Krasnoselskikh, V., Le Guirriec, E., et al.: Early results from the Whisper instrument on Cluster: an overview, Ann. Geophys., 19, 1241-1258, 2001, http://www.ann-geophys.net/19/1241/2001/. 
Dubinin, E., Skalsky, A., Song, P., Savin, S., Kozyra, J., Moore, T. E., Russell, C. T., Chandler, M. O., Fedorov, A., Avanov, L., Sauvaud, J.-A., and Friedel, R. H. W." Polar-Interball coordinated observations of plasma and magnetic field characteristics in the regions of the northern and southern distant cusps, J. Geophys. Res., 107(A5), 1053, doi:10.1029/2001JA900068, 2002.

Dunlop, M. W., Cargill, P., Stubbs, T., and Woolliams, P.: The high altitude Cusps: HEOS-2, J. Geophys. Res., 105, 27 509-27 517, 2000.

Dunlop, M. W., Lavraud, B., Cargill, P., Taylor, M. G. G. T, Balogh A., et al.: Cluster observations of the cusp: magnetic structure and dynamics, Surveys in Geophys., 26, 95-113, 2005.

Eastman, T. E., Boardsen, S. A., Chen, S.-H., and Fung, S. F.: Configuration of high-latitude and high-altitude boundary layers, J. Geophys. Res., 105, 23 193-23 238, 2000.

Farrell, W. M. and Van Allen, J. A.: Observations of the Earth's polar cleft at large radial distances with the Hawkeye 1 satellite, J. Geophys. Res., 95, 20 945-20 958, 1990.

Fedorov, A., Dubinin, E., Song, P., Budnick, E., Larson, P., and Sauvaud, J.-A.: Charac-teristics of the exterior cusp for steady southward interplanetary magnetic field: Interball observations, J. Geophys. Res., 105, 15 945-15 957, 2000.

Frank, L. A.: Plasma in the earth's polar magnetosphere, J. Geophys. Res., 76, 5202-5219, 1971.

Fritz, T. A., Chen, J., and Siscoe, G. L.: Energetic ions, large diamagnetic cavities, and Chapman-Ferraro cusp, J. Geophys. Res., 108(A1), 1028, doi:10.1029/2002JA009476, 2003.

Genot, V., Louarn, P., and Mottez, F.: Alfvén wave interaction with inhomogeneous plasmas: acceleration and energy cascade towards small-scales, Ann. Geophys., 22, 2081-2096, 2004, http://www.ann-geophys.net/22/2081/2004/.

Grande, M., Fennell, J., Livi, S., Kellett, B., Perry, C. H., Anderson, P., Roeder, J., Spence, H., Fritz, T., and Wilken, B.: First Polar and 1995-034 Observations of the mid-Altitude Cusp During a Persistent Northward IMF Condition, Geophys. Res. Lett., 24, 1475-1478, 1997.

Gustafsson, G., André, M., Carozzi, T., Eriksson, A. I., Fälthammar, C.-G., Grard, R., et al.: First results of electric field and density observations by Cluster EFW based on initial months of operation, Ann. Geophys., 19, 1219-1240, 2001, http://www.ann-geophys.net/19/1219/2001/.

Haaland, S. E., Sonnerup, B. U. Ö., Dunlop, M. W., Balogh, A., Georgescu, E., Hasegawa, H., Klecker, B., Paschmann, G., PuhlQuinn, P., Rème, H., Vaith, H., and Vaivads A.: Four-spacecraft determination of magnetopause orientation, motion and thickness: comparison with results from single-spacecraft methods, Ann. Geophys., 22, 1347-1365, 2004, http://www.ann-geophys.net/22/1347/2004/.

Haerendel, G.: Microscopic plasma processes related to reconnection, J. Atmos. Terr. Phys., 40, 343-353, 1978

Haerendel, G., Paschmann, G., Sckopke, N., and Rosenbauer, H.: The Frontside Boundary Layer of the Magnetosphere and the Problem of Reconnection, J. Geophys. Res., 83, 3195-3216, 1978.

Johnstone, A. D., Alsop, C., Burge, S., Carter, P. J., Coates, A. J., Coker, A. J., Fazakerley, A. N., Grande, M., Gowen, R. A., Gurgiolo, C., Hancock, B. K., Narheim, B., Preece, A., Sheather, P. H., Winningham, J. D., Woodliffe, R. D.: Peace: A plasma electron and current experiment, Space Sci. Rev., 79, 351-398, 1997.

Kessel, R. L., Chen, S. H., Green, J. L., Fung, S. F., Boardsen, S. A., Tan, L. C., Eastman, T. E., Craven, J. D., and Frank, L. A.: Evidence of high-latitude reconnection during northward IMF: Hawkeye observations, Geophys. Res. Lett., 23, 583-586, 1996.

Klimov, S. I., Nozrdrachev M. N., Triska P.,Vojta J., Savin S., et al.: Investigation of plasma waves by combined wave diagnostic device BUDWAR PROGNOZ-10-INTERCOSMOS, Cosmic Research (Transl. from Russian), 24, 177-184, 1986.

Lavraud, B., Dunlop, M. W., Phan, T. D., Rème, H., Bosqued, J. M., Dandouras, I., Sauvaud, J. A., Lundin, R., Taylor, M. G. G. T., Cargill, P. J., Mazelle, C., Escoubet, C. P., Carlson, C. W., McFadden, J. P., Parks, G. K., Moebius, E., Kistler, L. M., Bavassano-Cattaneo, M. B., Korth, A., Klecker, B., and Balogh, A.: Cluster observations of the exterior cusp and its surrounding boundaries under northward IMF, Geophys. Res. Lett., 29, 20, 1995, doi:10.1029/2002GL015464, 2002.

Lavraud, B., Rème, H., Dunlop, M. W., Bosqued, J. M., Dandouras, I., Sauvaud, J.-A., Keiling, A., Phan, T. D., Lundin, R., Cargill, P., Escoubet, C. P., Carlson, C. W., McFadden, J. P., Parks, G. K., Moebius, E., Kistler, L., Amata, E., Bavassano-Cattaneo, M.B., Korth, A., Klecker, B. and Balogh, A.: Cluster observes the high altitude/exterior cusp regions, Surveys in Geophys., 26, 13, 135-175, 2005.

Maynard, N. C.: Coupling of the solar-wind/IMF to the ionosphere through the high latitude cusps, Surveys in Geophys., 26, 1-3, 255-280, 2005.

Mozer, F. S., Phan, T. D., and Bale, S. D.: The complex structure of the reconnecting magnetopause, Phys. Plasmas, 10, 2480, doi:10.1063/1.1570419, 2003.

Newell, P. T. and Meng, C. I.: Ionospheric projections of magnetospheric regions under low and high solar wind pressure conditions, J. Geophys. Res., 99, 273-286, 1994.

Nikutowski, B., Buchner, J., Wiechen, H., Auster, U., Fornacon, K. H., Rustenbach, J., Klimov, S., and Savin, S.: A highlatitude boundary layer - crossing INTERBALL measurements and MHD model results, Adv. Space Res., 22, N1, 161-165, 1998.

Nikutovski, B., Buechner, J., Klimov, S., Petrukovich, A., Romanov, S., and Savin, S.: INTERBALL observations of field aligned current signatures due to collisionless reconnection, in: VIIth International Conference on Plasma Astrophysics and Space Physics, edited by: Buchner, J., Axford, I., Marsch, E., Vasyliunas, V., Kluwer AP, Dordrecht, 687-692, 1999.

Paschmann, G., Haerendel, G., Sckopke, N., and Rosenbauer, H.: Plasma and magnetic field characteristics of the distant polar cusp near local noon: The entry layer, J. Geophys. Res., 81, 2883-2899, 1976.

Phan, T., Frey, H. U., Frey, S., Peticolas, L., Fuselier, S., Carlson, C., Rème, H., Bosqued, J.-M., Balogh, A., Dunlop, M., Kistler, L., Mouikis, C., Dandouras, I., Sauvaud, J.-A., Mende, S., McFadden, J., Parks, G., Moebius, E., Klecker, B., Paschmann, G., Fujimoto, M., Petrinec, S., Marcucci, F., Korth, A., and Lundin, R.: Simultaneous Cluster and IMAGE observations of cusp reconnection and auroral proton spot for northward IMF, Geophys Res. Lett., 30, 1509, doi:10.1029/2003GL016885, 2003.

Rème, H., Aoustin, C., Bosqued, M., Dandouras, I., Lavraud, B., Sauvaud, J. A., et al.: First multispacecraft ion measurements in 
and near the Earth's magnetosphere with the identical Cluster ion spectrometry (CIS) experiment, Ann. Geophys., 19, 1303-1354, 2001 , http://www.ann-geophys.net/19/1303/2001/.

Russell, C. T.: POLAR eyes in the cusp, Proc. of the Cluster II workshop: Multiscale/Multi-point Plasma Measurements, ESA SP-449, 47-55, 2000.

Savin, S. P., Borodkova, N. L., Budnik, E. Yu., Fedorov, A. O., Klimov, S. I., et al.: Interball tail probe measurements in outer cusp and boundary layers, in: Geospace Mass and Energy Flow: Results from the International Solar-Terrestrial Physics Program, edited by: Horwitz, J. L., Gallagher D. L., and Peterson, W. K., American Geophysical Union, Washington D.C., Geophys. Monogr., 104, 25-44, 1998.

Savin, S. P., Zelenyi, L. M., Romanov, S. A., Klimov, S. I., Skalsky, A. A., et al.: Turbulent Boundary layer at the Border of Geomagnetic Trap, JETP Lett., 74, 11, 547-551, 2001.

Savin, S., Buechner, J., Consolini, G., Nikutowski, B., Zelenyi, L., Amata, E., et al.: On the properties of turbulent boundary layer over polar cusps, Nonlin. Processes Geophys., 9, 443-451, 2002, http://www.nonlin-processes-geophys.net/9/443/2002/.

Savin, S. P., Zelenyi, L. M., Amata, E., et al.: Dynanic Interaction of Plasma Flow with Hot Boundary Layer of Geomagnetic Trap, JETP Lett., 79, 452-456, 2004a.

Savin, S., Zelenyi, L., Romanov, S., Sandahl, I., Pickett, J., Amata, E., et al.: Magnetosheath - Cusp Interface, Ann. Geophys., 22, 183-212, 2004b.

Savin, S., Skalsky, A., Zelenyi, L. Song, P., Fritz, T. A., Amata, E., Buechner, J. Blecki, J., et al.: Magnetosheath interaction with high latitude magnetopause, Surveys in Geophys., 26, 5$55,2005 \mathrm{a}$.

Savin, S., Zelenyi, L., Amata, E., Buechner, J., Blecki, J., Greco, A., Klimov, S., Lopez, R. E., Nikutowski, B., Panov, E., et al.: Magnetosheath interaction with high latitude magnetopause: dynamic flow chaotization, Planet. Space Sci., 53, 133-140, 2005 b.

Savin, S., Amata, E., Andre, M., Dunlop, M., Khotyaintsev, Y., et al.: Experimental study of nonlinear interaction of plasma flow with charged thin current sheets: 2. Hall dynamics, mass and momentum transfer, Nonlin. Processes Geophys., 13, 377-392, 2006 (cited as $[\mathrm{S}])$

Scudder, J. D., Mozer, F. S., Maynard, N. C., and Russell, C. T.: Fingerprints of collision-less reconnection at the separator, I, Ambipolar-Hall signatures, J. Geophys. Res., 107(A10), 1294, doi:10.1029/2001JA000126, 2002.

Sibeck, D. G., Paschmann, G., Treumann, R. A., Fuselier, S., Lennartsson, A., et al.: Plasma Transfer Processes at the Magnetopause, Space Sci. Rev., 88, 207-283, 1999.
Silin, I. and Buechner, J.: Nonlinear instability of thin current sheets in antiparallel and guided magnetic fields, Phys. Plasma, 10, 9, 1-10, 2003.

Song, P., Russell, C. T., Gombosi, T. I., Spreiter, J. R., Stahara, S. S., and Zhang, X. X.: On the processes in the terrestrial magnetosheath, 1: Scheme development, J. Geophys. Res., 104, 22 345-22 356, 1999.

Stasiewicz, K.: Finite Larmor radius effects in the magnetosphere, Space Sci. Rev., 65, 221-253, 1994.

Syrovatskii, S. I.: Formation of current sheets in a plasma with a frozen-in strong magnetic field, Sov. Phys. JETP, 33, 933-940, 1971.

Taktakishvili, A., Greco, A., Zimbardo, G., Veltri, P., Cimino, G., Zelenyi, L., and Lopez, R. E.: The penetration of ions into the magnetosphere through the magnetopause turbulent current sheet, Ann. Geophys., 21, 1965-973, 2003, http://www.ann-geophys.net/21/1965/2003/.

Vaisberg, O. L., Galeev, A. A., Zeleny, L. M., Zastenker, G. N.,Omelchenko, A. N., Klimov, S. I., Savin, S. P., et al.: Fine structureof the magnetopause from the measurements of the satellites PROGNOZ-7 and PROGNOZ-8, Cosmic Research, (Transl. from Russian), 21, 49-55, 1983.

Vaivads, A., Khotyaintsev, Y., André, M., Retinò, A., Buchert, S. C., Rogers, B. N., Décréau, P., Paschmann, G., Phan, T. D.: Structure of the Magnetic Reconnection Diffusion Region from Four-Spacecraft Observations, Phys. Rev. Lett., 93, 10, 105001, doi:10.1103/PhysRevLett.93.105001, 2004.

Van Allen, J. A. and Adnan, J.: Observed currents on the earth's high-latitude magnetopause, J. Geophys. Res., 97, 6381-6395, 1992.

Woch, J. and Lundin, R.: Magnetosheath plasma precipitation in the polar cusp and its control BY the interplanetary magnetic field, J. Geophys. Res., 97, 1421-1430, 1992.

Yamauchi, M., Nilsson, H., Eliasson, L., Norberg, O., Boehm, M., Clemmons, J. H., Lepping, R. P., Blomberg, L., Ohtani, S.-I., Yamamoto, T., Mukai, T., Terasawa, T., and Kokubun, S.: Dynamic response of the cusp morphology to the solar wind: A case study during passage of the solar wind plasma cloud on February 21, 1994, J. Geophys. Res. ,101, 24 675-24 687, 1996.

Zelenyi, L. M., Delcourt, D. C., Malova, H. V., and Sharma, A. S.: "Aging" of the magnetotail thin current sheets, Geophys. Res. Lett., 29, 12, 1608, doi:10.1029/2001GL013789, 2002. 